\title{
The Application Research of Genetic Algorithm
}

\author{
Jumei Zhang \\ Weinan Normal University, Weinan, Shaanxi, 714000
}

Keywords: Genetic Algorithm, Application Research, Geophysical Prospecting

\begin{abstract}
Genetic algorithm is now an optimization method widely used in geophysical prospecting. This paper summarizes the basic principle and method of genetic algorithm and its developing direction. It analyzes the implement process of the method, and introduces the genetic process, the current situation and the application prospect of the genetic algorithm.
\end{abstract}

\section{Introduction}

Genetic Algorithm (GA) was proposed by American scholar Holland in 1975. It is a kind of optimization and efficient search algorithm that simulates the theory of natural selection and genetics based on the survival of the fittest. Because of its strong ability to solve problems and its wide adaptability, it has penetrated into various fields of research and engineering in recent years and achieved good results. Genetic algorithm is a kind of random global search algorithm, which searches the target space randomly. It treats possible solutions in the problem domain as an individual or chromosome of a population and encodes each individual in binary notation or floating-point notation to achieve parameterization of the model, One by one mapped to the chromosome space chromosomes, repeated genetic groups based on the operation, according to the predetermined objective function of each individual to be evaluated through the basic genetic operation process, and repeated iterative optimization of breeding to produce new Generation, continue to get a better group, while the global parallel search to search for the best individuals in the optimization group, to obtain the optimal solution to meet the requirements.

\section{Genetic algorithm concept and calculation method}

Chromosome: When using GA, the problem needs to be solved into a fixed symbol string, each of which represents a gene. A chromosome represents a solution to a problem. Each chromosome is called an individual. Population (Population): the total number of chromosomes generated per generation. A group contains a collection of some solutions to the problem in this generation. (3) Fitness: Each individual corresponds to a solution to a specific problem. The function value corresponding to each solution is the fitness function. It is an indicator to measure the fitness of the chromosome to the environment and an objective function that reflects the actual problem.

The process of solving with GA is based on the parameter set of the problem to be solved, a population is generated randomly, the fitness function and the selection rate are calculated, and genetic operations such as selection, crossover and mutation are performed. If the iterative convergence condition is satisfied, the population is the best Individuals, otherwise, the generation of a new generation of groups to re-genetic operation, reciprocating cycle until the conditions are met.

The basic genetic operations are: Select, according to a certain probability from the previous generation to select $\mathrm{M}$ individuals as parents, directly copied to the next generation, the chromosome does not change. One of the most common and simplest choice probabilities is: Ps (xi) $=\mathrm{f}(\mathrm{xi}) /$ Eif (xi) where $\mathrm{f}(\mathrm{xi})$ is the fitness of the model xi. Crossover (Crossover) is the process of randomly selecting two individuals from the old population, exchanging genetic information and generating offspring. Mutation (Mutation) That is, the process of generating new genes, select the group of individuals (chromosomes), random selection of a bit to be inverted operation. This can prevent the genetic algorithm converges to the local optimal solution, help to expand the scope of 
optimization, and enhance search capabilities. Especially in the latter part of the genetic algorithm, when the individuals and fitness values in the population are similar, the further evolution of the population depends on the mutation operation. In summary, the process of finding the optimal solution using genetic algorithm can be divided into the following steps: 1) coding the problem to be solved; 2) randomly giving a set of initial solutions $X(0)=(X 1, X 2, \ldots, X n)$; (3) evaluating the performance of the current group, and calculating fitness $\mathrm{f}$ (xi) for each individual $\mathrm{x}$ in the current group $\mathrm{x}(\mathrm{t})$; selecting a certain number of solutions from the current solution as the objects of the genetic operation according to the evaluation result of; genetic operation of the selected solution to obtain a new set of solutions; return to evaluate the new solution; if the current solution to meet the requirements or evolutionary process reaches a certain value, the calculation is completed, or continue. Genetic algorithm in the first few iterations, the emergence of individuals are good and bad coexist, the fitness is not high, with the increase of the number of iterations, individuals with high fitness were followed by genetic. Genetic algorithms have many advantages for solving optimization problems, such as selection of conditions. Especially, GA has high search order and search has exploratory and self-evolving capabilities.

\section{GA application status and prospects}

Genetic algorithm is the result of multidisciplinary integration and infiltration, and has developed into a self-organizing and adaptive integrated technology. As an effective global search method, it has been widely used in many fields including engineering design, manufacturing, artificial intelligence, computer science, bioengineering, petroleum exploration, automatic control, social science, commerce and finance. Oil exploration is mainly used in many fields such as predicting oilfield production, optimizing oilfield development, optimizing logging interpretation, and inferring the distribution of formation permeability. Genetic algorithms make up for the lack of traditional optimization techniques, and show its characteristics and attractiveness in the solution and application of many problems in the field of oil and gas exploration and development. However, at present, there are still some shortcomings and deficiencies in both theory and application of genetic algorithms. In practice, genetic algorithms are often prone to premature convergence and poor convergence, and genetic algorithms are not universal, In terms of problem solving, they can not completely replace the existing optimization techniques in a particular field and they all have their own scope of application. For a particular area, genetic algorithms often can not compare with algorithms that deal with problems in this area.

The reactive power optimization compensation problem in power system is a multivariable and multi-constrained mixed nonlinear programming problem. The manipulated variables include continuous variables (such as node voltage and generator reactive power), and discrete variables Load tap changer tap gear, compensation capacitor switching group), making the optimization process is very complicated. However, the traditional linear programming method, non-linear programming method and mixed integer programming method, which are applied to reactive power optimization compensation, have the problems of approximate processing of discrete variables and do not meet the actual requirements of reactive power planning. Therefore, However, there are still some common limitations that are easy to fall into the local extreme area and can not achieve the accurate processing of discrete variables. Because of its own characteristics, genetic algorithm is especially suitable for solving the problem of multi-objective hybrid optimization and has stable convergence characteristics. It is an advanced global optimization method. Reactive power optimization is a set of initial solutions under the condition of power system. The genetic algorithm is limited by various constraints, and its advantages and disadvantages are evaluated by the fitness function. The low fitness value is abandoned. Only the high fitness value Have the opportunity to iterate their characteristics to the next round of solutions, through the selection, crossover, mutation and other genetic operations, the population gradually tends to be the best, and finally get the optimal solution. The disadvantage of simple genetic algorithm is that it takes a long time to compute and can easily converge to the local extreme if the population and the genetic algebra are not large enough. For this reason, an improved genetic algorithm, or a combination of genetic 
algorithm and other algorithms, is proposed to improve computational speed and computational accuracy. In this paper, annealing genetic algorithm is used in combination with simulated annealing selection algorithm, that is to use annealing selection as an individual replacement strategy to avoid falling into the local optimal solution. The first part, reactive optimization algorithm has been given earlier. The other part, that is, the goal of real-time control system of reactive power and voltage, is to use EMS application software to remotely control the transformer taps and capacitor banks in the substation in the regional dispatch center so as to realize the safe and economic operation of the regional power grid. As a real-time control system, not only to ensure good enough optimal solution, but also to ensure optimal solution corresponding to the control of the program's implementation. Therefore, in addition to solving the problems of theory and algorithm, a large number of technical problems in project implementation should also be solved, including handling of constraints, starting methods, dead zone handling, safety considerations and program implementation.

\section{Evolutionary algorithm in genetic algorithm to solve the path optimization problem specific methods}

Identify a specific set of locations to visit and call a certain number of vehicles from the warehouse to access these locations. So how to choose is the optimal path of the vehicle so that the vehicles can access each position in a certain order, not only satisfying specific constraints, but also minimizing the time and the optimal path of the vehicle. I propose an improved genetic algorithm, the genetic algorithm coding mechanism to natural number coding mechanism, the advantages of this mechanism is very intuitive. Re-use three replica championship approach to evolutionary solution. Experiments show that the improved algorithm can better solve the optimal path

The new coding scheme adopted makes coding very straightforward and responds to problems of all sizes. And to set up a special position, as a genetic position. The order of visiting a specific location is the required solution (including infeasible solutions). Each locus has its own constraints that need to be met. Such as location, time of arrival, etc. For example, if the solution of the problem is: $1: 0 \rightarrow 2 \rightarrow 6 \rightarrow 5 \rightarrow 0$ Route $2: 0 \rightarrow 8 \rightarrow 3 \rightarrow 7 \rightarrow 1 \rightarrow 0$ When looking for the optimal solution, the head of the position to be passed by the vehicle And the tail connected together, so that the chromosome can be encoded as 256173849 . The disadvantage of doing so is the lack of a route demarcation point, but later it is more convenient to cross the operation. As long as the optimal solution in decoding, in accordance with the order to insert the node to the route, you can solve it. If you can not meet some time or can not satisfy other constraints, you can insert a new path from the insertion point. According to the evolutionary thinking, when the route is optimal, the total cost and time of the visit will be reduced. Therefore, as far as possible, the location will be inserted into the original route during the evolutionary process.

Through the improved genetic algorithm, we can solve the problem of finding the optimal path in the process of enterprise, postal delivery and freeway picking. Other industries on the optimal path of the problem can be solved. Studying the genetic algorithm can help to improve the profits of the related enterprises, and the enterprises can also improve the better service for the customers, and the demands of the people can also be met to the greatest extent. Therefore, the promotion and application of the algorithm is very meaningful.

\section{Conclusion}

The basic theory of the genetic algorithm is weak, there are many problems need to explore improvements in practice, its continuous development in recent years and its successful application in many areas has shown its unique advantages, with the genetic algorithm to further improve And its development and application in the field of oil and gas exploration and development, its extensive potential for application will be further tapped and its scope of application will continue to expand. This will provide a powerful tool for solving some oil exploration and development problems. 


\section{Acknowledgements}

Shaanxi Provincial Department of Education 2017 Special Scientific Research Program (17JK0259)

Weinan Teachers College International Cooperation Project on Education Science (17GJHZ17)

Weinan Teachers College 2016 Humanities and Social Science Project (16SYB15)

\section{References}

[1] Jin Cong.Heuristic Genetic Algorithm and Its Application [J]. Journal of Numerical Computing and Computer Applications. 2003 (01)

[2] Yang Xiaohua, Lu Guihua, Yang Zhifeng, Li Jianqiang. Gray code accelerated genetic algorithm and its theoretical research [J]. Systems Engineering Theory and Practice. 2003 (03)

[3] Zhuang Jian, Wang Sunan.Study on self-adjusting genetic algorithm [J]. Journal of System Simulation. 2003 (02)

[4] Chen Xiaoping, Yu Shenglin. Improvement of the Cross Strategy of Real Genetic Algorithms [J] .Journal of Electronics \& Information Technology. 2003 (01)

[5] Wen Xinchao, Zhou Linxia, Niu Kai.A genetic algorithm based on hybrid coding [J] .Electronic Technology, 2003 (01)

[6] Chen Chao, Chen Jialian, Yu Feng. Using bit manipulation of a genetic algorithm program [J] .Application Research of Computers. 2002 (11) 